\title{
La Révolution française et son influence \\ sur la chirurgie dentaire \\ en Amérique
}

\section{RÉSUMÉ}

Éric CURTIS

D.D.S.

2010 West 16 th Street

Safford

Arizona 85546

États-Unis.

La Révolution française, en créant un climat d'insécurité, favorisa l'émigration de nombreux professionnels parmi les meilleurs de leur profession, en particulier des médecins et des dentistes, vers les États-Unis. Ils y ont trouvé une terre d'accueil en plein développement, où commençait à s'organiser la dentisterie en tant que telle avec la création d'écoles, de revues et de sociétés scientifiques.

Le Comité de Rédaction a estimé que certains articles historiques méritaient une republication étant donné leur intérêt, ce qui est le cas de cet article paru en décembre 1992 et que nous proposons à nouveau à nos lecteurs.

- émigration

- profession dentaire

- Révolution française 
$>$

Antoine Lavoisier monta les marches de la guillotine le 8 mai 1794. Il est reconnu comme le fondateur de la chimie moderne. II nomma l'oxygène et décrivit son rôle dans la combustion. II démontra la loi de conservation de la matière et écrivit la première équation chimique. Il fut à l'origine du concept d'élément chimique. Mais le citoyen Lavoisier, désabudé par la Révolution française qu'il avait servie comme membre de la Commune de Paris et administrateur de la Trésorerie nationale, fut déclaré coupable pour avoir travaillé dans le gouvernement royal comme collecteur de taxes, il fut exécuté le jour même de sa condamnation. De l'exécution de Lavoisier, le procureur public déclara: "La République n'a pas besoin des savants» [1]. En 1989, la France commémora le bicentenaire du début de sa révolution tumultueuse. Parallèlement aux festivités, il y eut une grande réflexion sur le premier soulèvement populaire des temps modernes. La révolution américaine introduisit la notion de liberté et de gouvernement du peuple dans un monde de monarchies. Mais les concepteurs de la Constitution américaine étaient des colons qui voulaient simplement transférer l'administration de la patrie anglaise à eux-mêmes. Ils cherchaient la stabilité au lieu du bouleversement. Ils essayaient de maintenir un ordre au lieu d'encourager le changement. La Révolution française, de toutes façons, fut un cataclysme social. En déposant leur propre souverain, les Français annulèrent le droit divin des rois, et leur lutte idéaliste devint celle qui transformerait une société entière.

La confusion qui s'ensuivit eut de profonds effets sur l'évolution de la médecine dentaire moderne. À cause de la convulsion politique qui régnait en France, la profession dentaire naissante chercha un centre d'intérêt vers les États-Unis, où la dentisterie était déjà bien éta- blie comme une véritable profession autonome.

À travers l'histoire, la France a maintenu une formidable réputation concernant les idées et les inventions. En science et en médecine, particulièrement, elle a beaucoup contribué au progrès du monde. Les premiers hôpitaux occidentaux se développèrent en France: I'Hôtel-Dieu de Paris fut fondé au vile siècle. Au XIVe siècle, le chirurgien Guy de Chauliac modela la pensée médicale européenne avec son traité Chirurgia Magna, écrit en 1363, définissant les critères de la chirurgie pour des siècles. Chauliac identifia la dentisterie comme une catégorie séparée des soins de santé, inventant le terme issu du latin, dentista, «celui qui soigne les dents».

Ambroise Paré, chirurgien du XVIIe siècle, est considéré comme le fondateur de la chirurgie moderne. II n'était pas médecin, et avait acquis ses connaissances par l'apprentissage et l'expérience. II fut successivement barbier, chirurgien-barbier et maître chirurgien-barbier. Cette évolution montre clairement la hiérarchie socio-médicale de cette époque. En ce qui concerne les soins dentaires, le traitement des patients pourrait se diviser ainsi : les médecins ayant étudié la théorie et le latin donnaient les consultations sur les maladies buccales. Un médecin de nos jours explique : «La médecine, à l'origine, était surtout parlée et comportait très peu de diagnostics et de traitement». Les extractions étant une forme de la chirurgie, étaient mises entre les mains des guérisseurs, de chirurgiens ou des barbiers-chirurgiens, qui occupaient un rang social inférieur aux médecins (physiciens). L'art prothétique était l'apanage des artisans. Les compétitions entre forains ou charlatans - qui extrayaient ou cassaient des dents pour amuser les passants étaient habituelles. 


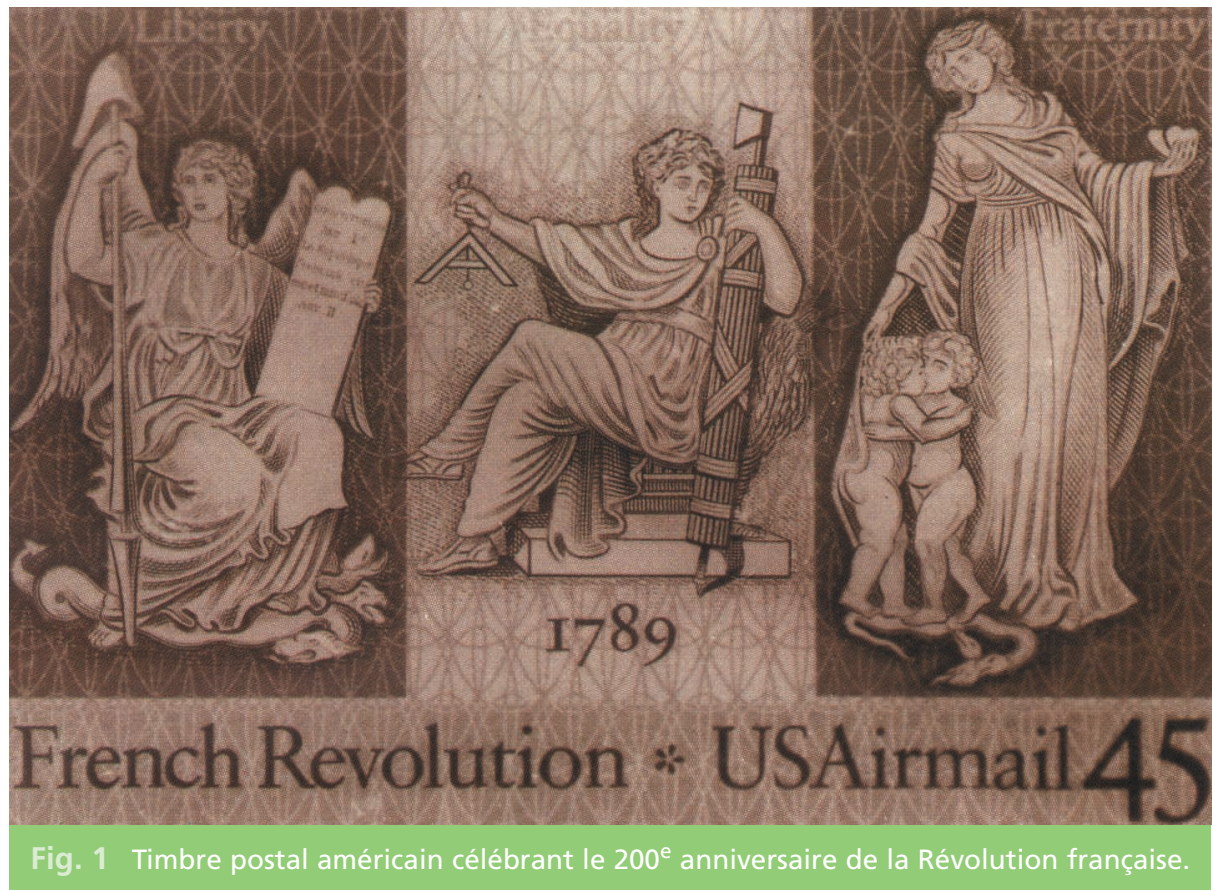

Paré devint chirurgien au service de plusieurs rois de France. Sa réputation et ses ouvrages, qui comprenaient de vastes descriptions d'instruments et de techniques dentaires, commencèrent à faire progresser la chirurgie artisanale vers l'art médical.

En 1619, probablement sous la pression des guildes chirurgicales qui cherchaient à augmenter leur statut officiel, une loi qui concéda un rang égal aux dentistes, guérisseurs d'os et lithotomistes fut édictée en France. Après le passage d'un examen devant une commission de trois maîtres en chirurgie, chaque spécialiste était considéré comme expert dans sa branche de chirurgie.

Les chirurgiens parisiens du Collège de SaintCôme, affirmant leur indépendance par rapport aux orgueilleux médecins de I'Université de Paris, mirent un frein à la pratique de la chirurgie mineure par les charlatans. En 1699, le roi Louis XIV décréta que la formation des chirurgiens-dentistes devait être faite au Collège.
Après un cursus d'étude de deux ans, un étudiant pouvait être appelé «chirurgien-dentiste». Le nom, devenu "dentist» en anglais, est le terme encore utilisé pour désigner les dentistes en France.

La prééminence française dans la chirurgie dentaire au XVIII siècle est surtout due à un seul homme, Pierre Fauchard, qui sortit la profession de l'artisanat et créa le concept moderne de la dentisterie. Fauchard n'a pas inventé la dentisterie, mais il catalogua les techniques, procédures et idées existantes, et il les publia à travers le monde à une époque où le secret entre praticiens était considéré comme essentiel pour les affaires. Son chef-d'œuvre, Le chirurgien-dentiste ou Traité des dents, marqua le commencement de l'information partagée entre praticiens. Fauchard affirma que la dentisterie était déficiente dans trois domaines clefs. II dénonça le manque de littérature dentaire, les rares opportunités de s'instruire, et les vagues règlements sur la pratique à travers la 


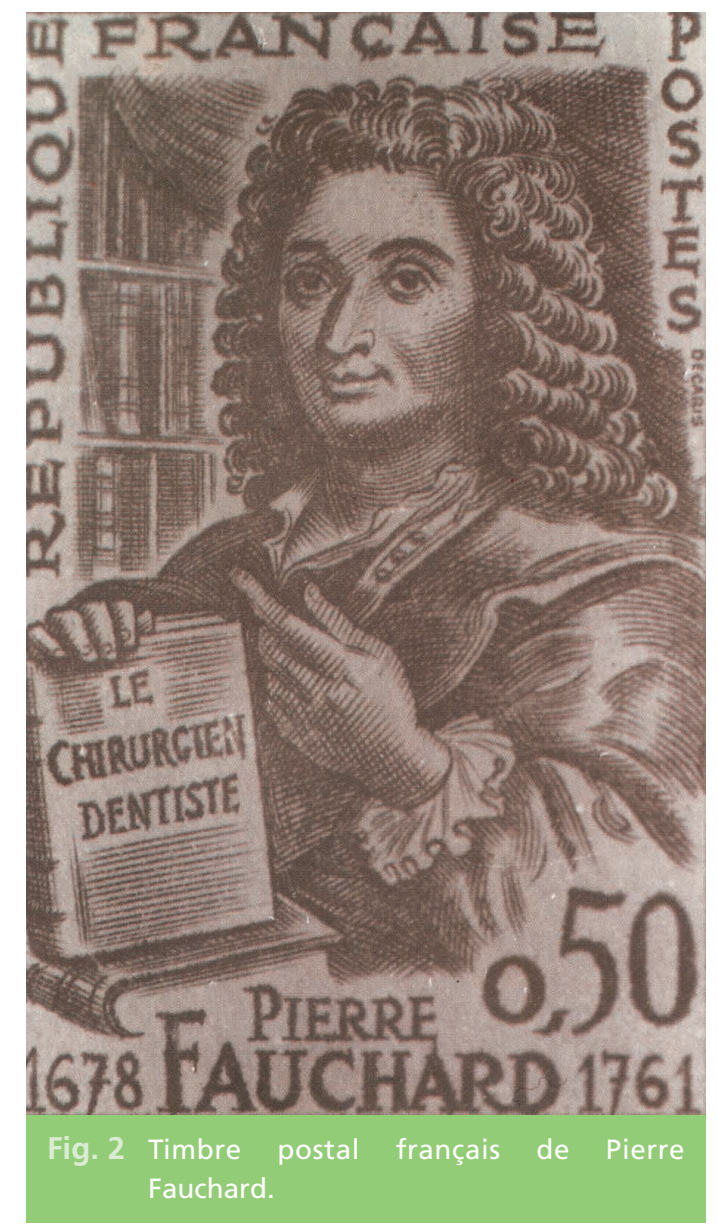

France. II s'éleva avec force et éloquence contre "des gens sans théorie et sans expérience, qui s'en sont emparés, et la pratiquent au hasard, n'ayant ni principes, ni méthodes».

Les enseignements de Fauchard prospérèrent. La philosophie de la dentisterie fut réformée par la génération suivante de chirurgiens-dentistes. Les dentistes français acquirent une réputation de supériorité.

Pendant qu'un renouveau des connaissances humanistes se développait en Italie, la France s'enfonçait dans une époque d'absolutisme politique. L'autorité monarchique augmenta régulièrement du XVe jusqu'au XVIIIe siècle. Le château de Versailles devint la vitrine de la noblesse affaiblie et Louis XIV put se vanter:
«L'État c'est moi». Les philosophes français soutinrent que les idées autocratiques prédominant dans le monde faisaient s'affaiblir la pensée innovatrice. Descartes avait proclamé que le doute systématique et le raisonnement logique étaient les bases de la vraie connaissance. Ses enseignements présagèrent le Siècle des Lumières, le mouvement du XVIII siècle qui insistait sur la raison et le progrès humain.

Une obsession pour les affaires saisit la France, et les distinctions de classe s'estompèrent sous l'influence égalisante de l'argent. C'était une époque où le chimiste Lavoisier pouvait aussi diriger une perception. Le Siècle des Lumières, en donnant une importance à l'amélioration de la société, influença la Révolution française. Un historien révisionniste l'interprète comme une passion pour la modernité, qui n'a pas commencé au niveau du prolétariat, mais en haut de la société, chez les nobles, les avocats et les prêtres. Les tensions sociales augmentèrent pendant que les élites discutaient pour savoir comment mieux ressusciter le pays. On essayait de justifier la violence au nom de la réforme sociale. Les rêves de grandes possibilités s'évanouirent, et les factions rivales exploitèrent la rage des victimes du changement, les ménagères sans pain, les artisans sans travail. La Terreur effaça tout sens des buts communs et le progrès espéré s'arrêta.

La France se redressa, et ses savants en médecine commencèrent à innover. En 1820, PierreJoseph Pelletier isola la quinine de l'arbre tropical Cinchona. Bientôt, avec d'autres scientifiques, il isola la morphine, la caféine et la digitaline. En 1829, le pharmacien Henri Leroux parvint à extraire la salicine de l'écorce du saule. Louis Pasteur créa pratiquement la microbiologie: son Institut est toujours un centre majeur de recherches biomédicales. 
La médecine progresse souvent pendant les guerres. Les urgences absolues dues aux blessures peuvent amener à une improvisation qui conduit à l'innovation. L'expérience et la discipline du champ de bataille ont forcé les chirurgiens français à rompre avec leurs habitudes, donnant un essor aux profonds changements à venir dans la pratique et l'enseignement de la médecine. La guerre qui commença en 1792 fournit aux médecins une éducation différente de l'éducation traditionnelle, qui cessa d'exister, et l'on peut dire que le champ de bataille devint leur école de médecine.

Pour la dentisterie, la Révolution française fut un désastre. La vision de Fauchard d'un corps de praticiens dentaires autonomes, spécialisé et instruit, fut l'idéal révolutionnaire de l'égalité. En essayant d'atteindre l'égalité parmi le peuple, les anciennes méthodes d'instruction furent abandonnées. En 1792, toutes les exigences pour la formation professionnelle furent annulées. Seule une licence disponible à tout citoyen, qui en faisait la demande, était nécessaire pour l'exercice de la médecine ou de la chirurgie. Le chaos qui s'ensuivit atteignit tous les domaines de la médecine. Les charlatans de foire surgirent de partout, incontrôlés et non sanctionnés par l'État.

Il devint évident que l'exercice médical sans règles conduisait à des situations dangereuses. En 1795, la Convention nationale rétablit l'enseignement médical théorique. En 1789, les représentants du peuple furent envoyés à Paris pour conférer sur les aspects de la vie française. Les minutes de ces assemblées laissent apparaître de fréquentes requêtes pour éliminer les charlatans, pour fournir plus de médecins dans les zones rurales et édicter des lois appropriées pour les médecins et les chirurgiens [4].
En 1803, Napoléon fit rétablir les lois sur la profession médicale. Étrangement, les dentistes n'étaient pas concernés ; il n'y eut aucun texte légal régissant la chirurgie dentaire avant 1892. Des dentistes itinérants exerçant en plein air existaient encore à Paris jusqu'au $x x^{e}$ siècle. Pendant tout le $X I x^{e}$ siècle, la dentisterie était la seule profession en France à ne pas posséder ses règles, dans un pays «où tout est réglé» [5]. La dentisterie se discrédita.

Le centre d'évolution de la dentisterie émigra vers les États-Unis [6]. En premier lieu, le tumulte de la Révolution française interrompit le progrès scientifique et brisa la stabilité sociale nécessaire à la nourriture de la naissante et fragile profession. En second lieu, les États-Unis, jeunes et avides de légitimité, développaient un esprit ambitieux, curieux et entreprenant. Ils constituaient un climat idéal pour des professionnels et des commerçants, et les médecins européens y trouvèrent une grande demande pour leurs compétences. La guerre révolutionnaire américaine ne fut pas seulement le tremplin de la Révolution française, mais elle encouragea le transfert des talents français vers les États-Unis. Les spécialistes ont répondu que peu de dentistes avaient fui la Révolution, mais beaucoup de gens instruits pratiquant l'art dentaire étaient probablement des chirurgiens, dont quelque 300 quittèrent la France.

Parmi les meilleurs dentistes ayant émigré dans les anciennes colonies anglaises, se trouvait Jacques Gardette (1756-1831), un chirurgien de la Marine française qui avait été détaché auprès des armées de Lafayette. Gardette fut l'auteur du premier article scientifique sur la dentisterie dans la littérature américaine et I'on pense qu'il a formé le premier dentiste né aux États-Unis à avoir connu la célébrité : Josiah Flagg. 
Un autre dentiste américain éminent né en France fut Jean-Pierre Le Mayeur (mort en 1806). Celui-ci arriva à New York, occupée par les Anglais, pendant la révolution américaine. Une déclaration anti-française faite en sa présence le rendit furieux et lui fit quitter la ville. Il fut invité au quartier général de George Washington qui devint son patient.

Le troisième facteur qui a contribué à l'augmentation de la prééminence de la chirurgie dentaire en Amérique est le développement de la technologie. L'accroissement de la population et l'arrivée de la révolution industrielle firent surgir les espoirs d'une vie meilleure. II en résulta une montée de la consommation qui encouragea les expériences des inventeurs et des manufacturiers. Beaucoup d'entre eux appliquèrent leur ingéniosité à la dentisterie. Le développement dans les années 1850 du caoutchouc vulcanisé par Charles Goodyear, par exemple, signifiait que les prothèses dentaires pouvaient être réalisées plus aisément et à un meilleur coût. Tout à coup, ces prothèses furent accessibles au plus grand nombre.

Quatrièmement, la progression du nombre des écoles primaires gratuites produisit un nombre croissant de lecteurs, ce qui eut pour conséquence la prolifération du matériel de lecture. En ce qui concerne la dentisterie, cela signifie une augmentation de l'impression et de la circulation de brochures pour l'éducation des patients et de publications pour les professionnels. Dans les jeunes États-Unis, les praticiens formés en Europe et ceux nés en Amérique firent de grands progrès. N'étant pas entravés par une tradition restrictive comme les corporations européennes de chirurgiens-barbiers et aidés par une population développant connaissances et discernement, les dentistes en Amérique étaient libres de s'organiser en profession. Vers 1826, un dentiste originaire
d'Allemagne, Léonard Koecker, qui avait exercé à Londres et à Philadelphie, disait de l'Amérique : «Nulle part au monde l'art dentaire n'a atteint un pareil niveau» [6].

Comme Pierre Fauchard l'avait annoncé, la dentisterie, en tant que profession, dépend de trois éléments reposant sur une base solide: l'enseignement, l'organisation et les publications. Pendant les années 1839-1840, cette trilogie apparut pour la première fois au monde aux États-Unis. Pour une grande part, l'impulsion vint de deux hommes: Horace Hayden et Chapin Harris.

Hayden et Harris organisèrent la première société dentaire (la Société américaine de chirurgie-dentaire) et le premier journal dentaire, American Journal of Dental Science. Ils furent les fondateurs du Baltimore College of Dental Surgery, la première école destinée à l'enseignement dentaire ; ils firent partie des quatre professeurs qui donnèrent des cours d'anatomie, physiologie, pharmacologie, thérapeutique et de "dentisterie appliquée». Le cursus durait deux ans, équivalent en cela aux écoles de médecine de l'époque. Les docteurs Hayden et Harris créèrent le diplôme de "Chirurgia Dentium doctoris» dont les initiales furent reprises dans le sigle D.D.S. (Doctor in Dental Surgery). Par l'attribution de ce diplôme, les dentistes devinrent des «Docteurs».

Ainsi, les principes furent élaborés et les institutions fondées pour l'ascension de la chirurgie dentaire aux États-Unis comme une profession distincte de la médecine. La France, héritière des plus grands progrès médicaux d'Europe, perdit sa prééminence dans la dentisterie pendant la tourmente de la Révolution; ce qui fut bénéfique à la médecine ne le fut pas pour la dentisterie. En bouleversant le milieu social et scientifique favorable au progrès de cette profession embryonnaire, la France laissa échapper ses meilleurs dentistes, 
sa réputation sur le plan dentaire et les idées de ses innovateurs sur le plan médical et dentaire au profit de l'Amérique.

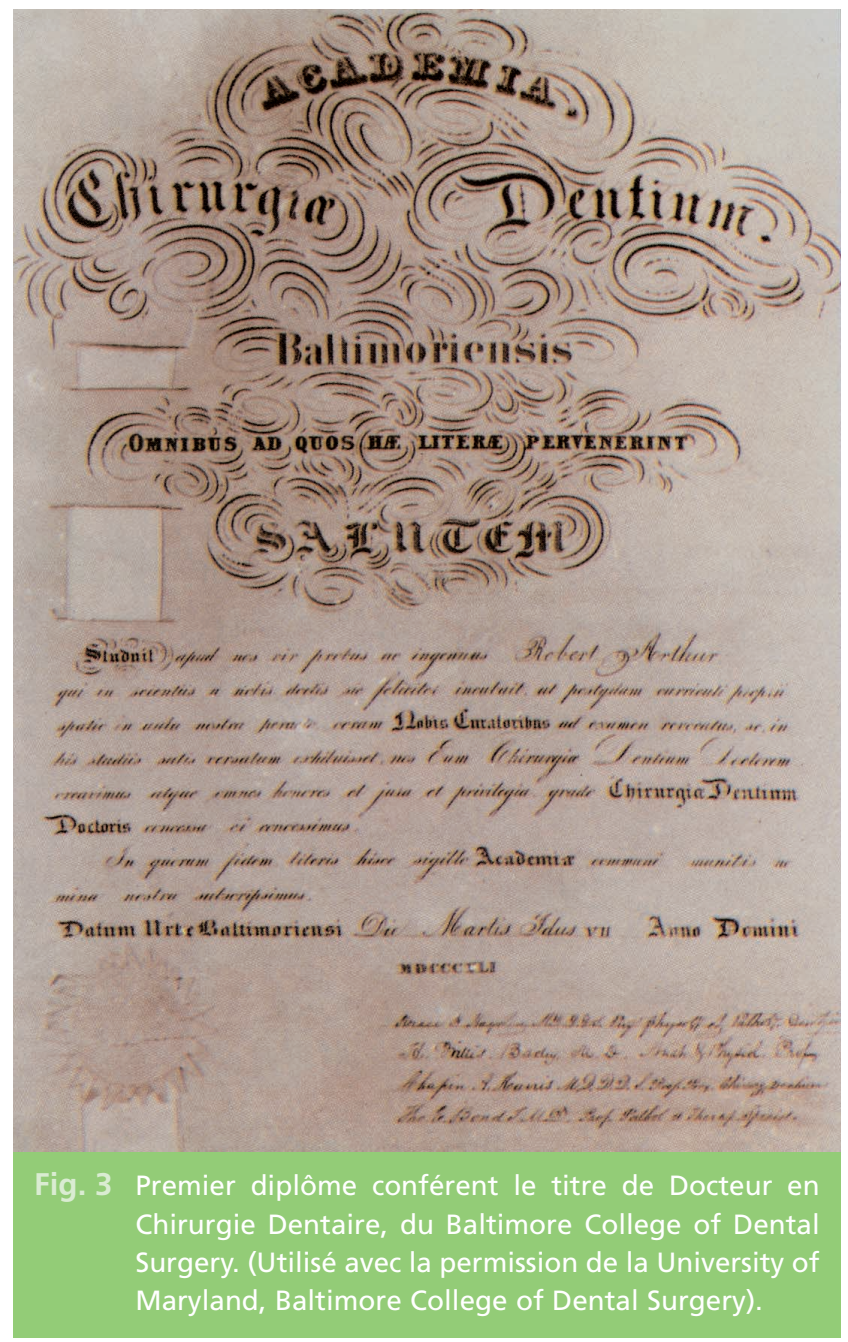

Thomas Evans, le dentiste de Philadelphie qui exerçait à Paris au XIXe siècle, devint le praticien de la Cour de Napoléon III. Dans sa jeunesse, il avait dit «qu'en tant qu'Américain, il ne deviendrait jamais un excellent dentiste». En réfléchissant sur sa carrière, il médita : "Tout cela a subi un bouleversement étrange, car c'est l'Amérique qui a fait cette profession... qui l'a sortie de l'artisanat où elle se trouvait reléguée en France lorsque j'y habitais» [5].

Au XIXe siècle, la dentisterie prospéra dans le Nouveau Continent: la chirurgie buccale et l'orthodontie devinrent des spécialités, les équipements furent développés, I'anesthésie devint une réalité. Les Américains tel Thomas Evans trouvèrent leur apogée en retournant en Europe après 1840, renversant ainsi I'habituelle influence culturelle entre les continents. La dentisterie actuelle doit sa forme à la pensée scientifique et aux conditions politiques dans les deux pays. Les historiens de France et des États-Unis s'influencent les uns et les autres au cours des profonds changements de leurs révolutions respectives. La tradition française d'excellence médicale et la pensée du Siècle des Lumières engendrèrent les principes de I'exercice dentaire moderne. La société ouverte de l'Amérique permit la réalisation de ces idées.

\section{Bibliographie}

1. Gooding J, Dorozynski A. Audacity: 200 years of french innovation. Supplement. Amer. Heritage 1989;40(5):1-24.
2. Schama S. Citizens: a chronicle of the french Revolution. New York, Knopf, édit., 1989.

3. Vess DM. Medical revolution in France, 1789-1796.

Gainesville, Florida State University Press, édit., 1975.

4. Caron P. 1789 : les cahiers de doléances des médecins 
et chirurgiens.

Chir Dent de France 1990;60(521):20-26.

5. Carson G.

The dentist and the empress.

Boston, Houghton Mifflin, édit., 1983.

6. Ring ME.

An illustrated history.

New York, Abrams, édit.

7. Plusieurs éducateurs aux États-Unis voulaient faire de la dentisterie une partie de la médecine américaine (voir Gevitz N. Autonomous profession or medical speciality:

the stomatological

movement and american

dentistry.

Bull Hist Med

1988;62:407-428).

Le fait que la chirurgie

dentaire n'ai jamais été

incorporée

dans la médecine

peut être une mise

en évidence de la résistance

à la spécialisation de la part

des pouvoirs médicaux.

La dentisterie américaine

devint en effet

la première spécialité

à plein temps

de la médecine, et la première

dont l'Amérique

put s'enorgueillir,

voir Shryock RS.

Medicine and society

in America: 1660-1860.

Ithica, Cornell University

Presss, édit., 1975.

8. Quand Harvard University organisa une école dentaire en 1867 , le titre décerné fut celui de Doctor of Dental Medicine, ou Docteur en Médecine Dentaire, abrégé en D.M.D.

Ainsi, il existe actuellement deux titres dentaires aux États-Unis.

\section{SUMMARY}

\section{The French Revolution and its influence on dental surgery in America}

Éric. CURTIS

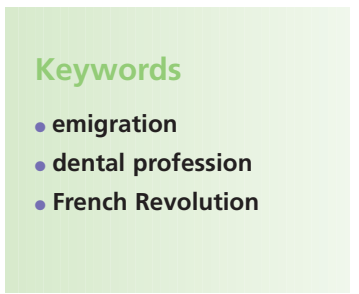

The French Revolution created a climate of insecurity which led to the emigration to the United States of many professionals, some of the best in their fields, and in particular doctors and dentists. There they found a receptive terrain in full expansion, where dentistry as such was becoming organized, with the creation of schools and scientific journals. 